\title{
Line Loss Calculation for Distribution network Based on States Characterization
}

\author{
Junming HUANG \\ School of Electric Power Engineering, South China University of Technology, Guangzhou 510640,
China
}

Keywords: distribution network ; power loss calculation; state characterization; three-phase unbalance; distributed power generation.

\begin{abstract}
A novel real-time line loss calculation method based on state characterization is proposed for calculating the line loss of distribution network in this paper. First, the load currents collected by the measuring terminals during a certain period are characterized, such that the change characteristics of electric loads are reflected fully, and the pressure of data transmission and data processing is relieved effectively. Second, based on the state characterizations, the line loss of distribution network is calculated by the split phase calculation method, resulting to the solution of the problems about the three-phase unbalance and the accessing of distributed power generation. Finally, the proposed method is applied to a real distribution network, and the results prove its effectiveness well.
\end{abstract}

\section{Introduction}

Line loss rate is an important economic and technical index to reflect the operation condition of the power system. In order to manage the line loss of distribution network and improve its economy, it is necessary to compute the theoretical line loss of the distribution network for accurately [1-2]。

Now , more challenges were proposed in distribution line loss calculation field . On the one hand, with the intensive growth of power load, unbalanced load of three-phase increased in distribution network [3].On the other hand, with the increasing of distributed power, the power flow of distribution network is more complex[4]。 More difficulties are made by these changes to the distribution network loss calculation。

The calculation methods can not well meet the requirements of accurate calculation for distribution network losses at present. The existing distribution network losses calculation methods can be classified into two kinds of two: estimation algorithm and power flow algorithm [5-8]。The estimation algorithm includes RMS current method, average current method, equivalent resistance method. These algorithms need simple operation and less data, but can not reflect the change process of state power, which results in a relatively large calculating error. In addition, situations like three-phase load balance or DG are not considered in the algorithms, so it can not adapt to the new requirements of the distribution network line loss calculation. Power flow algorithm can get a more accurate line loss of the time section, but distribution network power flow calculation is more prone to convergence problems than main power grid.

Considering the unbalanced and access of distributed power conditions, the convergence of distribution network power flow calculation is lower, the application of this algorithm is largely limited the new situation of the calculation.

In recent years, more and more real-time measuring devices are installed in load nodes for better observation of distribution network, which provides an important condition for the accurate calculation of line loss. In this case, a calculation algorithm was proposed in [9] ,based on the data offeeder terminal unit (FTU), which greatly improves the accuracy of line loss calculation; At the same time [10-12] using the data of feeder terminal unit and distribution transformer terminal unit (TTU) to calculate line loss, which also achieved good results. But the two algorithms require intensive transmission of a large number of measurement terminal data to main station, the data transmission and processing burden is too large ,on the condition that the communication network in the distribution network is not perfect enough. [13] obtain the real-time line loss of the distribution network by the state estimation based on terminal data, but this method still exists the problem of 
excessive burden of data transmission, and the convergence problem of state estimation in distribution network.

In this paper, a new distribution network loss calculation method is proposed base on the idea of process state characterization. The load data collected by the measuring terminals are characterized, and these characteristic quantities were used for loss calculation by phases, whici provides a new way to calculate distribution network line loss in three-phase unbalanced or DG case.

\section{The Basic Definition of the Process State Characterization}

With the development of smart metering terminal, millisecond acquisition of state data is realized in distribution network already. But the current distribution network communication facilities are far worse than the main grid, so it is difficult to meet the requirements of data transmission of a large number of intensive collection. To solve this problem, some scholars put forward the analysis algorithm of time process oriented[14-17]。

The basic idea of time process oriented analysis algorithm is refining the variation of the power system in a period of time, to characterize the state change of power system in the period. Assume that in a given time $\mathrm{T}$, the running state of the system an evaluation index $\mathrm{Z}$ can be expressed as functional form of the state variables of the system $\mathrm{X}(\mathrm{t})$ :

$$
Z=\int_{0}^{T} f[X(t)] \mathrm{d} t
$$

And can find a specific state variable $\tilde{X}$, Convert $\mathrm{Z}$ to $\tilde{X}$ function:

$$
Z=T \cdot g(\tilde{X})
$$

Then $\tilde{X}$ is the the characteristic state variable of $\mathrm{Z}$, the calculating process is called process state characterization[18]。By the method, a time process oriented complex problem can be converted into a relatively simple problem oriented features, thus the the quantity of transmission data processing reduce effectively.

\section{Process State Characterization of Line Loss Calculation}

State characteristic current based on intelligent metering terminal. The line losses of distribution network can be regarded as the integral of power loss $\Delta P_{\text {loss }}(t)$ at unit time of the time

$$
\Delta A=\int_{0}^{T} \Delta P_{\text {loss }}(t) \mathrm{d} t
$$

In the formula, $\Delta A$ for the line loss of the distribution network.

According to the definition of the process state characterization, if a specific current $\tilde{I}$ can be found in at the time of $T$, of which the loss is equal to the actual loss the same period of time, which is

$$
\Delta A=T \cdot g(\tilde{I})
$$

In the formula, $g(\tilde{I})$ is a function of time independent.

The current can characterize the change of the line loss in this period of time, and $\tilde{I}$ is called the loss state characteristics of current.

According to equation (3) and (4), $\tilde{I}$ must meet the current state of line loss:

$\int_{0}^{T} I_{t}^{2} R d t=T \times \tilde{I}^{2} \times R$

Thus, the loss characteristics for current $\tilde{I}$ can be expressed as : 


$$
\tilde{I}=\sqrt{\int_{0}^{T} I_{t}^{2} \mathrm{~d} t / T}
$$

Most of the current intelligent metering terminal has function of storage and calculation,so feature processing of load current state can be carried out in situ measurement terminal, then the data is uploaded to the main station for line loss calculation, which can reduce much pressure of measurement data transmission and centralized processing. However , the measurement terminal is installed in the load side, only characteristics of branch line can be obtained. We need to calculate the current state characteristics of trunk level by the state characteristic current of branch line.

According to the KCL, the current phase of a line is equal to the sum of the currents of all the branches at the next level

In formula (6) , the characteristic current of only contains the information of the current effective value, without the information of current phasor, so it is necessary to construct a complex state characteristic current.

$I_{\mathrm{p}, m}$ is defined as the real part of phase current, $I_{\mathrm{q}, m}$ is defined as the imaginary part of phase current.

$$
\left\{\begin{array}{l}
I_{\mathrm{p}, m}=I_{m} \cos \theta_{m} \\
I_{\mathrm{q}, m}=I_{m} \sin \theta_{m}
\end{array} \quad m=\mathrm{A}, \mathrm{B}, \mathrm{C}\right.
$$

In the formula, $I_{m}$ for amplitude phase current; $\theta_{m}$ for the current phase angle.

According to the formula (6), the real part and the imaginary part of the three-phase current are processed by the state characteristics.

$$
\left\{\begin{array}{l}
\tilde{I}_{\mathrm{p}, m}=\operatorname{sign}\left(\cos \theta_{m}\right) \cdot \sqrt{\int_{0}^{T} I_{\mathrm{p}, m}^{2} \mathrm{~d} t / T} \\
\tilde{I}_{\mathrm{q}, m}=\operatorname{sign}\left(\sin \theta_{m}\right) \cdot \sqrt{\int_{0}^{T} I_{\mathrm{q}, m}^{2} \mathrm{~d} t / T}
\end{array} \quad m=\mathrm{A}, \mathrm{B}, \mathrm{C}\right.
$$

In the formula , $\tilde{I}_{\mathrm{p}, \mathrm{m}}$ for the real part of loss state characteristic current; $\tilde{I}_{\mathrm{q}, \mathrm{m}}$ for the imaginary part of the line loss state characteristics of current. The sign function is used to determine the direction of the state characteristics current, so as to distinguish ordinary load and DG situation. The state characteristic current common load branch is positive, meanwhile, the state characteristic current distributed power supply branch is negative.

Thus, it is possible to obtain the state characteristics current in the complex form of each phase in a period of time

$$
\tilde{I}_{m}=\tilde{I}_{\mathrm{p}, m}+\mathrm{j} \tilde{I}_{\mathrm{q}, m} \quad m=\mathrm{A}, \mathrm{B}, \mathrm{C}
$$

Using KCL, the characteristics current of upper sections branch can be obtained.

\section{Calculation of Distribution Line Loss}

Loss of transmission lines. State current characteristics can be obtained by the process state characterization method. On this basis, we can further calculate the sections of line loss. Considering the three-phase unbalanced situation is more serious, the method by phase calculation is used :

$$
\left\{\begin{array}{l}
\Delta W_{\mathrm{LA}, i}=\sum_{j=1}^{N}\left|\tilde{I}_{\mathrm{A}, i, j}\right|^{2} R_{\mathrm{A}, i} T \\
\Delta W_{\mathrm{LB}, i}=\sum_{j=1}^{N}\left|\tilde{I}_{\mathrm{B}, i, j}\right|^{2} R_{\mathrm{B}, i} T \\
\Delta W_{\mathrm{LC}, i}=\sum_{j=1}^{N}\left|\tilde{I}_{\mathrm{C}, i, j}\right|^{2} R_{\mathrm{C}, i} T
\end{array}\right.
$$


In the formula, $\Delta W_{\mathrm{LA}, i} 、 \Delta W_{\mathrm{LB}, i} 、 \Delta W_{\mathrm{LC}, i}$ for three phase loss of line $i ; R_{\mathrm{A}, i} 、 R_{\mathrm{B}, i} 、 R_{\mathrm{C}, i}$ for three phase resistance the line $i$; $\tilde{I}_{\mathrm{A}, i, j} 、 \tilde{I}_{\mathrm{B}, i, j} 、 \tilde{I}_{\mathrm{C}, i, j}$ for three phase state characteristic current of line ${ }^{i}$ in the period of $j$; $\mathrm{n}$ for the total time of characterization ; T for each time interval.

The total loss of the line is the sum of three phases loss :

$$
\Delta W_{\mathrm{L}, i}=\Delta W_{\mathrm{LA}, i}+\Delta W_{\mathrm{LB}, i}+\Delta W_{\mathrm{LC}, i}
$$

Loss of transformers. In the case of three phase unbalance, the variable loss of the public transformer can be calculated by the state characteristic current of the winding

$$
\Delta W_{\mathrm{Cu}, i}=\sum_{j=1}^{n} \frac{\Delta P_{\mathrm{f}, i}}{3} \times\left[\left(\frac{\left|\tilde{I}_{\mathrm{A}, i, j}\right|}{I_{\mathrm{N}, i}}\right)^{2}+\left(\frac{\left|\tilde{I}_{\mathrm{B}, i, j}\right|}{I_{\mathrm{N}, i}}\right)^{2}+\left(\frac{\left|\tilde{I}_{\mathrm{C}, i, j}\right|}{I_{\mathrm{N}, i}}\right)^{2}\right] T
$$

In the formula, $\Delta W_{\mathrm{Cu}, i}$ for variable loss of public transformer $i ; \Delta P_{\mathrm{f}, i}$ for short-circuit loss of public transformer $i ; I_{\mathrm{N}, i}$ for the amplitude of rated current of public transformer.

The fixed loss of public transformer is

$$
\Delta W_{\mathrm{Fe}, i}=\Delta P_{0, i} \cdot n T
$$

In the formula, $\Delta W_{\mathrm{Fe}, i}$ for the fixed loss of public transformer ; $\Delta P_{0, i}$ for the loss of public transformer without load.

The total loss of public transformer is

$$
\Delta W_{\mathrm{T}, i}=\Delta W_{\mathrm{Cu}, i}+\Delta W_{\mathrm{Fe}, i}
$$

The total loss of distribution network. The total loss of the distribution network is the sum of the losses of all the lines in the distribution network

$$
\Delta W=\sum_{i=1}^{M} \Delta W_{\mathrm{L}, i}+\sum_{i=1}^{N} \Delta W_{\mathrm{T}, i}
$$

In the formula, $\mathrm{M}$ is the total number of lines in the distribution network, and $\mathrm{N}$ is the total number of public transformers.

\section{Example Analysis}

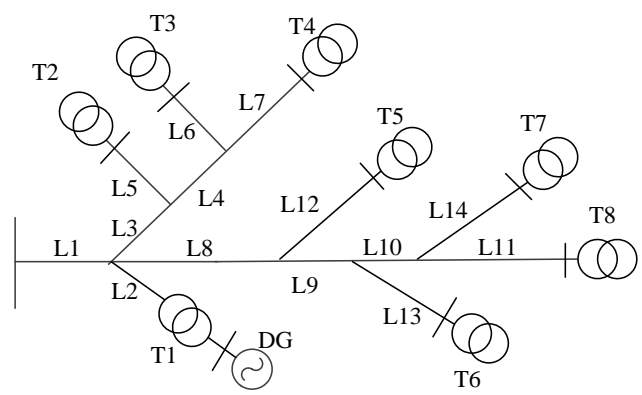

Fig. 1 Connection of pilot

A $10 \mathrm{kV}$ power distribution network in Dongguan is used as the indication point verify the effectiveness of the proposed method in the case of distributed power supply. The network connection of point is shown in Figure 1. T1 branch is connected with a capacity of $1250 \mathrm{~kW}$ of small hydropower, T2, T3, T6, T8 for the public transformer, T4, T5, T7 for the special transformer.

Figure 2 shows loss curve of one day, and table 1-2 shows loss in line and public transformer in the point, table 3 shows the calculation results of proposed method and the equivalent resistance method, and statistical line loss. The calculation results show that the calculation method proposed in this paper is also applicable in the distributed power distribution network line loss calculation. 


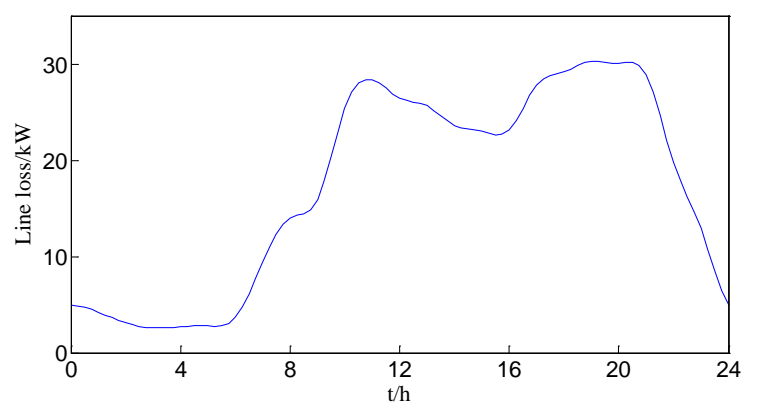

Fig. 2 Line loss curve of indication pilot

Tab. 1 Power loss of transmission I ines of the pilot $(\mathrm{kW} \cdot \mathrm{h})$

\begin{tabular}{cccccc}
\hline Line & Loss & Line & Loss & Line & Loss \\
\hline L1 & 23.55 & L6 & 0.89 & L11 & 2.44 \\
L2 & 3.64 & L7 & 1.19 & L12 & 0.66 \\
L3 & L8 & L13 & 1.15 \\
L4 & L9 & L10 & 12.87 & L14 & 0.81 \\
L5 & 0.15 & 6.07 & & \\
\hline
\end{tabular}

Tab. 2 Power loss of transformers of the pilot $(\mathrm{kW} \cdot \mathrm{h})$

\begin{tabular}{cccc}
\hline Transformer & Variable loss & Fixed loss & Total loss \\
\hline T2 & 5.29 & 16.20 & 24.49 \\
T3 & 31.95 & 41.04 & 72.99 \\
T6 & 41.26 & 42.48 & 83.76 \\
T8 & 87.65 & 50.16 & 137.81 \\
\hline
\end{tabular}

Tab. 3 Comparison of various methods in the pilot

\begin{tabular}{cccc}
\hline Algorithm & Algorithm in this paper & Statistical line loss & Equivalent resistance algorithm \\
\hline $\begin{array}{c}\text { Line loss } \\
/ \mathrm{kW} \cdot \mathrm{h}\end{array}$ & 397.91 & 406.78 & 373.83 \\
Line loss rate/\% & 3.14 & 3.21 & 2.95 \\
\hline
\end{tabular}

\section{Conclusion}

In this paper, a new method of distribution line loss calculation based on process state characterization is proposed:

In this paper, the proposed method fully considers the influence of the load change to the line loss, and significantly reduce the pressure of data transmission and processing, moreover, it has higher accuracy compared with the traditional method.

The method proposed in this paper can solve the problem of line loss calculation in the condition of unbalanced three-phase distribution, and is suitable for the calculation of line loss in the case of distributed power supply.

The proposed method is applied to a practical distribution network. The results show that the proposed method has good application effect and application value.

\section{References}

[1]LIU Jian, DUAN Jingjing. Line loss limitation analysis and optimal planning of loss reduction for distribution grids[J]. Power System Protection and Control, 2013,41(12): 27-35.

[2]CHEN Fang, ZHANG Li, HAN Xueshan, et al. Line loss evaluation and application in distribution network[J]. Power System Protection and Control, 2014, 42(13): 39- 44. 
[3]YANG Qiyu. Distribution network[M]. Beijing: China Electric Power Press,1998.

[4]WEN Xu, YAN Wei, HUANG Miao, et al. Probabilistic transmission loss rate evaluation under green energy generation uncertainty[J]. Power System Protection and Control, 2013,41(1):170-175.

[5]XU Shaoliang, SONG Ye, MIAO Zhumei, et al. Guide of caculator of grid energy loss.Bijing: China Electric Power Press, 2000.

[6]LI Peijie, LUO Cuiyun, BAI Xiaoqing, et al. An extended sequence components method for solving multiphase power flow in distribution networks [J].Power System Protection and Control,2016,44(3):15- 23.

[7]LIU Tinglei,WANG Shao, ZHANG Zhi, et al. Newton- Raphson method for theoretical line loss calculation of low-voltage distribution transformer district by using the load electrical energy[J]. Power System Protection and Control, 2015,43 (19): 143-148.

[8]HU Xiaosong, ZHANG Yin, XIE Guangbin, et al. A loop closing power flow calculation algorithm of distribution network with PV nodes[J]. Power System Protection and Control, 2015,43 (24):8-13.

[9]LIU Wei, LI Heng, SUN Fujie, et al. Topology analysis method for loss calculation of distribution network based on feeder sections and GIS[J]. Power System Technology, 2003,27(4):68-71.

[10]ZHU Faguo. Loss calculation method for distribution network with information from field terminal units[J]. Power System Technology, 2001,25(5):38-40.

[11]WANG Gangjun, WANG Chengmin, LI Heng, et al. Calculation method of theoretical network loss in power distribution network based on measured data[J]. Power System Technology, 2002,26(12):18-20.

[12]WANG Yonghua, WANG Zhengfeng. Research and application of power load measurement and on-Line power network loss compute based on SCADA/EMS system[J]. Power System Protection And Control, 2012,40(4):96-100.

[13]LI Bin, DU Mengyuan, WEI Wei, et al. Calculation of theoretical line loss based on quasi real-time data of smart distribution network[J]. Electric Power Automation Equipment, 2014,34(11):123-128.

[14]YIN Liyan, YU Jilai. Dynamic reconfiguration (DR) of distribution network with multi-time periods [J]. Proceedings of the CSEE, 2002,22(7):44-48.

[15]Golshan M E H, Arefifar S A. Distributed generation, reactive sources and network-configuration planning for power and energy loss reduction[J]. IEE ProceedingsGeneration, Transmission and Distribution, 2006,153(2): 127-136.

[16]LIU Wei, HAN Zhenxiang. Distribution system reconfiguration based on time interval[J]. Automation of Electric Power Systems,2006,30(10):33-38.

[17]FANG Xing, GUO Zhizhong, CAI Zhongqin. Distribution network dynamic reconfiguration based on time-interval [J]. Electric Power Automation Equipment, 2004,24(5):31-34.

[18]YANG Lin, GUO Zhizhong.System state characterization and application to distribution network reconfiguration[J]. Proceedings Of The CSEE, 2008,28 (16):39-44. 